\title{
Development of loop-mediated isothermal amplification (LAMP) assay combined with malachite green as a rapid screening test for Candidatus Mycoplasma haemominutum infection in cats
}

\author{
Sarunya Tedlongthong ${ }^{\mathrm{a}}$, Nareerat Viseshakul ${ }^{\mathrm{b}}$, Hirotomo Kato $^{\mathrm{c}, \mathrm{d}}$, Supatra Areekit ${ }^{\mathrm{e}, \mathrm{f}}$, \\ Somchai Santiwatanakul ${ }^{\mathrm{f}, g}$, Kosum Chansiri ${ }^{\mathrm{a}, \mathrm{f}, *}$ \\ ${ }^{a}$ Department of Biochemistry, Faculty of Medicine, Srinakharinwirot University, Bangkok 10110 Thailand \\ b Department of Pathology, Parasitology Unit, Faculty of Veterinary Science, Chulalongkorn University, \\ Bangkok 10330 Thailand \\ c Division of Medical Zoology, Department of Infection and Immunity, Jichi Medical University, Tochigi, \\ Japan \\ d Laboratory of Parasitology, Department of Disease Control, Graduate School of Veterinary Medicine, \\ Hokkaido University, Hokkaido, Japan \\ e Innovative Learning Centre, Srinakharinwirot University, Bangkok 10110 Thailand \\ f Centre of Excellence in Biosensors, Srinakharinwirot University, Bangkok 10110 Thailand \\ ${ }^{g}$ Department of Pathology, Faculty of Medicine, Srinakharinwirot University, Bangkok 10110 Thailand
}

*Corresponding author, e-mail: kchansiri@yahoo.com

\begin{abstract}
Feline infectious anaemia is caused by a Gram-negative, uncultivable, cell wall-deficient, epierythrocytic parasitic bacteria known as feline haemoplasmas (FHM) in the genus Mycoplasma, namely, Mycoplasma haemofelis (Mhf), Candidatus M. haemominutum (CMhm), and Ca. M. turicensis (CMtc). Here, a loop-mediated isothermal amplification (LAMP) targeting the 16S rRNA gene combined with malachite green (MG) based colorimetric assay was developed for the detection of feline CMhm infection. The limit of detection was determined using a ten-fold serial dilution of recombinant plasmid DNA (from $10^{8}$ to 1 copies). The result indicated that the LAMP-MG assay could detect as low as 264 copies corresponding to 264 organisms/ $\mu$ l of CMhm feline blood. Comparison between the LAMP-MG and standard polymerase chain reaction (PCR) surveying 105 clinical samples suggested that 17 and 15 samples were positive for CMhm, respectively. Validity of the LAMP-MG assay was assessed and calculated within $95 \%$ confidence intervals (CIs). The sensitivity, specificity, prevalence and accuracy were 100.0\%, 97.8\%, 14.3\%, and 98.1\%, respectively. The degree of agreement between LAMP-MG and standard PCR assays was $92.6 \%$, with a $\kappa$ coefficient of 1 (CI: 82.5-100.0\%). This LAMP-MG colorimetric assay may be applicable as a rapid screening point-ofcare testing for feline $\mathrm{CMhm}$, as well as for blood donors prior to blood transfusion by using unsophisticated equipment, such as a heating block or water bath. This technique could provide robust results, which are easily distinguished within $60 \mathrm{~min}$ after amplification.
\end{abstract}

KEYWORDS: molecular diagnosis, feline infectious anaemia, LAMP-MG assay, pH-sensitive indicator dye, low cost testing

\section{INTRODUCTION}

Feline infectious anaemia (FIA) is caused by Gramnegative, uncultivable, cell wall-deficient, epierythrocytic parasitic bacteria in the genus Mycoplasma known as feline haemoplasmas (FHM). The reported FHM are primarily Mycoplasma haemofelis (Mhf), Candidatus M. haemominutum (CMhm) and
Ca. M. turicensis $(\mathrm{CMtc})^{1-3}$. Destruction of red blood cells (RBCs) in cat patients occur by the attachment of the agents onto RBC surfaces resulting in direct damage to the RBC membranes. Then, the immune response called immune-mediated haemolytic anaemia is induced to destroy and eliminate the damaged RBCs from circulation primarily through phagocytosis in the spleen, 
liver, lungs and bone marrow. Furthermore, FHM varies in its pathogenicity from subclinical to lifethreatening haemolytic anaemia, associated with anorexia, lethargy, weight loss, hyperpyrexia, enlarged spleen, and sometimes sudden death within a few days if left untreated ${ }^{4-6}$.

$\mathrm{CMhm}$ is most commonly found in domestic and stray cats with the highest prevalence worldwide $^{7,8}$, including in Thailand ${ }^{9,10}$. CMhm infection can induce mild to moderate anaemia. Furthermore, $\mathrm{CMhm}$ co-infection with a retrovirus or other haemoplasma species may result in exacerbation of anaemia ${ }^{11}$. The infected feline carriers are potential reservoirs of the zoonotic pathogen, which is transmitted to other cats by aggressive interactions involving cat bites and scratches, and flea infestations ${ }^{12-14}$. However, the actual transmission route of haemoplasmas has not yet been established. Hence early detection and identification of pathogens are necessary for disease control in endemic areas.

The traditional method for the detection of FHM is limited to the level of microscopic examination of a stained blood smear. However, the sensitivity of the microscopic examination is less than $20 \%$ in chronically infected animals, and the specificity is often hampered by artefacts, such as stain precipitates and Howell-Jolly bodies ${ }^{15}$. Serological techniques, including immunoblotting, immunofluorescent antibody tests and recombinant protein-based ELISA have been reported ${ }^{16,17}$. Several polymerase chain reaction (PCR) assays, including conventional $\mathrm{PCR}^{18,19}$, multiplex $\mathrm{PCR}^{20}$, and real-time $\mathrm{PCR}^{21,22}$ have been developed as the reliable and sensitive diagnostic methods for the specific detection of FHM infection based on a highly specific region of the $16 \mathrm{~S}$ ribosomal RNA gene. However, conventional PCR and real-time PCR could limit their usages as pointof-care and field tests due to their time-consuming process and equipment dependence.

In 2000, Notomi et al developed a powerful innovative nucleic acid amplification method, loopmediated isothermal amplification (LAMP), which allows the amplification of DNA under isothermal conditions based on the auto-cycling strand displacement DNA synthesis by the Bst DNA polymerase, using a set of 4-6 primers that recognize specific regions of the target sequence ${ }^{23}$. LAMP produces extremely large amounts of amplified products by starting with loop-structure amplicons, followed by recycling and elongating processes, resulting in the production of various sized amplicons. This technique provides high specificity, sensitiv- ity, rapidity and enables simple visual detection by observing the turbidity, fluorescent dye and $\mathrm{pH}$ sensitive indicator dye. Although standard LAMPturbidity could detect LAMP products of white precipitate (magnesium pyrophosphate) by naked eye, it has a short constancy (5-10 s) after taking samples out either from the heating block or water bath ${ }^{24}$. This may need a real-time turbidimeter for more accurate results. According to LAMPfluorescent dyes assay, the use of a costly dye such as calcein or SYBR Green I, may need a UV illuminator for visual improvement. Furthermore, calcein which initially forms inclusion with manganese ion, could inhibit DNA polymerase activity and may decrease the overall sensitivity of the assay ${ }^{25}$. In this case, the post-addition of SYBR Green I can increase the risk of contamination. Even though, the colour indicator hydroxy naphthol blue has been reported for LAMP detection, the differentiation between positive and negative reaction colours as blue and purple are obscure, respectively.

Recently, the malachite green (MG), cationic triphenylmethane (or $\left[\mathrm{C}_{6} \mathrm{H}_{5} \mathrm{C}\left(\mathrm{C}_{6} \mathrm{H}_{4} \mathrm{~N}\left(\mathrm{CH}_{3}\right)_{2}\right)_{2}\right] \mathrm{Cl}$ ) dye has been successfully used as a $\mathrm{pH}$-sensitive indicator for visual end-point assessment of LAMP products $^{26-28}$. The colour change of MG (cationic form) depends on the $\mathrm{pH}$ of the solution $(\mathrm{pH}<2$ : yellow, $\mathrm{pH}$ 3-9: blue-green, $\mathrm{pH}>10$ : colourless) ${ }^{29}$. The absorption wavelength for MG is $621 \mathrm{~nm}$. In the LAMP-MG assay, positive and negative reactions are easily distinguished by naked eye as light blue and colourless, respectively. Pre-addition of MG do not interfere with the activity of the Bst DNA polymerase $^{30}$, eliminating the risk of contamination among samples. Furthermore, this technique can be used for the rapid screening of substantial number of samples with reproducible, robust and consistent results using simple and inexpensive equipment, such as a heating block or water bath under constant temperature. The advantages of LAMP-MG assay could improve and overcome the limitations from other LAMP detections as mentioned above. Hence the purpose of the present study was focused on the development of a rapid screening method for the detection of CMhm infection based on the 16S rRNA gene and to evaluate the diagnostic sensitivity and specificity from a total of 105 clinical samples. This method may be applicable for the control of CMhm by indicating the health condition of cats intended to be blood donors, particularly in asymptomatic carrier cats, subsequently preventing the unintentional infection of cats via blood transfusion. 


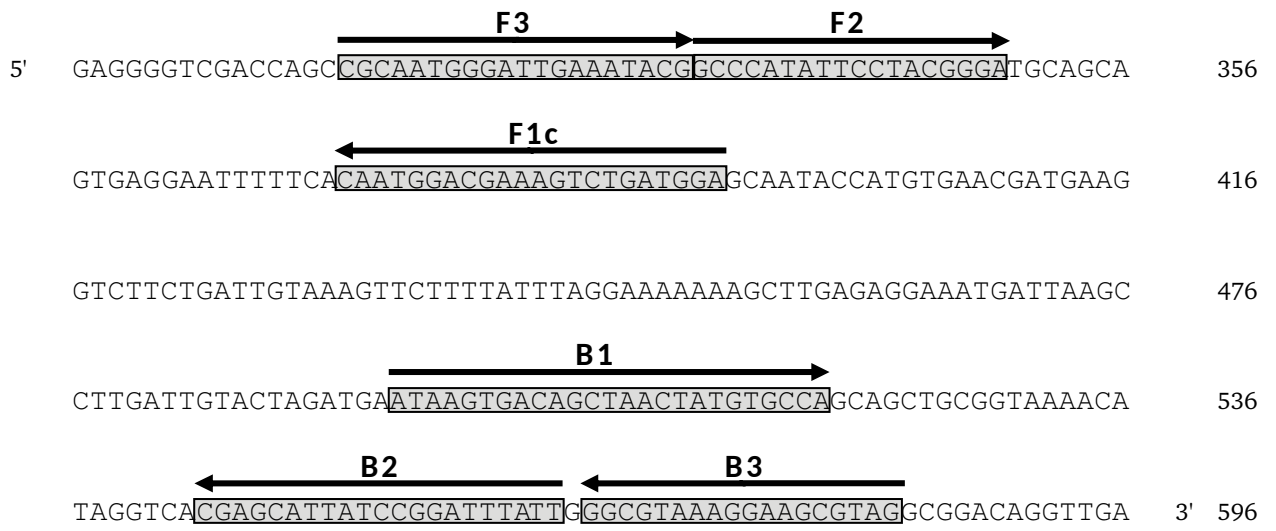

(Ca. M. haemominutum AY297712.1)

Fig. 1 Nucleotide sequence alignment of 16S rRNA genes of CMhm (AY297712.1). Each primer is indicated by arrows: forward outer primer (F3), backward outer primer (B3), forward inner primer (FIP; consists of F1c, a TTTT-linker and F2), and backward inner primer (BIP; consists of B1c, a TTTT-linker and B2).

\section{MATERIALS AND METHODS}

\section{Preparation of CMhm plasmid DNA template}

To prepare the CMhm plasmid DNA template used in this study, the specific PCR primers for cloning were designed based on a highly specific region of the 16S rRNA genes of feline CMhm (GenBank: AY297712.1). Then, CMhm genomic DNA was subsequently amplified by PCR, which produced a product $496 \mathrm{bp}$ in size. Once PCR was completed, the PCR product was purified and cloned into pGEM-T easy vector (Promega, USA), and brought to analytical process by restriction enzyme digestion and DNA sequencing (AIT Biotech, Singapore). Concentrations of recombinant plasmid DNA were measured with a NanoDrop 2000 UVVis spectrophotometer (Thermo Scientific, USA), and corresponding copy numbers were calculated as mole multiplies of Avogadro's number using an online program (http://cels.uri.edu/gsc/cndna.html), following the formula

$$
\text { Number of copies }=\frac{\text { Amount } \times 6.022 \times 10^{23}}{\text { Length } \times 10^{9} \times 650} \text {. }
$$

\section{LAMP primer design}

LAMP primers containing two outer primers; forward outer primer: F3 and backward outer primer: B3, and two inner primers; forward internal primer: FIP (consists of F1c, a TTTT-linker and F2) and backward internal primer: BIP (consists of B1c, a TTTT-linker and B2) were designed against a specific region of the 16S rRNA gene of feline CMhm
(GenBank: AY297712.1). The sequence was imported into the online PRIMEREXPLORER V4 program (http://primerexplorer.jp/elamp4.0.0/index. html) and synthesized by Greiner Bio-One, Japan (Fig. 1). This sequence was verified using the BLAST against the genus Mycoplasma and other species found in cat blood.

\section{LAMP assay}

The LAMP assay was conducted using the Loopamp DNA Amplification Kit (Eiken, Japan). The 15$\mu l$ reaction mixture consisted of $0.2 \mu \mathrm{M}$ of each outer primer (F3 and B3), $1.6 \mu \mathrm{M}$ of each inner primer (FIP and BIP), $1 \times$ reaction mix, $8 \mathrm{U}$ Bst DNA polymerase (Eiken, Japan) $1 \mu \mathrm{l}$ of CMhm plasmid DNA template $\left(10^{8}\right.$ copies), and the volume was adjusted by the addition of sterile distilled water. The optimum reaction temperature for the LAMP assay was tested at 61,63 , and $65^{\circ} \mathrm{C}$ for $60 \mathrm{~min}$ in a 96-well plate heating block (MiniT-100 incubator; Ningbo, China). The product size was analysed using 2.0\% agarose gel electrophoresis (AGE) stained with RedSafe Nucleic Acid Staining Solution (Intron Biotechnology, Korea). The LAMP products were observed under UV light using a UV-LED transilluminator (CUV 10; Clinx Science, China).

\section{Optimization of MG concentration}

The concentrations of MG in the LAMP reaction were $0.012 \%, 0.008 \%, 0.004 \%, 0.002 \%$, and $0.001 \%$. Each concentration was added into the reaction mixture and subsequently incubated at $63^{\circ} \mathrm{C}$ for $60 \mathrm{~min}$. The results were observed via naked 
eye based on a colour change of MG in the solution as light blue and colourless in positive and negative reactions, respectively.

\section{Limit of detection of LAMP-MG assay}

The limit of detection (LOD) of LAMP-MG assay was determined by using a ten-fold serial dilution of recombinant plasmid DNA containing the $\mathrm{CMhm}$ $16 \mathrm{~S}$ rRNA gene (from $10^{8}$ to 1 copies) in comparison to the LAMP-2.0\% AGE and standard LAMP-turbidity assay. Each experiment was done in triplicate. The final dilutions were calculated following the formula as mentioned above for the corresponding copy number of the 16S rRNA gene as a single copy gene $^{31}$.

\section{Clinical sample tests}

A total of 105 blood clinical samples used in this study were isolated from a pool of suspected cat patients with mild anaemia (haematocrit 15-25\%) registered at the Small Animal Teaching Hospital, Faculty of Veterinary Science, Chulalongkorn University, Thailand. Genomic DNA samples were extracted from EDTA preserved blood using the QIAamp DNA Blood Mini Kit (Qiagen, USA) and evaluated according to the manufacturer's instructions. The purity of DNA samples (A260/280) was determined using a NanoDrop 2000 UV-Vis spectrophotometer (Thermo Scientific, USA) and subsequently subjected to a molecular detection process. Once the LAMP-MG assay was completed, the positive results were re-confirmed by DNA sequencing using an ABI3130xl Genetic Analyser (Applied Biosystems, USA). Sequences obtained were analysed using the BIOEDIT and BLAST.

Standard PCR assay was performed for comparison of detection limit and clinical sample tests, using the same serial dilution and clinical sample genomic DNA conducted by the LAMP-MG assay. Concisely, the PCR reaction was carried out in a $25 \mu \mathrm{l}$ reaction mixture containing $0.4 \mu \mathrm{mol}$ of each primer (forward primer: 5'-ACGAAAGTCTGATGG AGCAATA- $3^{\prime}$ and reverse primer: 5'-ACGCCCAAT AAATCCGGATAAT $-3^{\prime}$, generating a 206 product of $193 \mathrm{bp}$ in size ${ }^{6,32}$ ), $0.25 \mathrm{mmol}$ of dNTPs, $3.5 \mathrm{mmol}$ of $\mathrm{MgCl}_{2}, 1 \times$ PCR buffer, $2.5 \mathrm{U}$ Taq polymerase (Invitrogen, USA), and $4 \mu \mathrm{l}$ of genomic DNA template $(10-100 \mathrm{ng} / \mu \mathrm{l})$. The reaction was performed under the following cycling parameters: initial denaturation at $94^{\circ} \mathrm{C}$ for $4 \mathrm{~min}$, followed by 35 cycles of denaturation at $94^{\circ} \mathrm{C}$ for $30 \mathrm{~s}$, annealing at $60^{\circ} \mathrm{C}$ for $30 \mathrm{~s}$, extension at $72{ }^{\circ} \mathrm{C}$ for $30 \mathrm{~s}$, and a final extension at $72{ }^{\circ} \mathrm{C}$ for $10 \mathrm{~min}$. Amplified products
Table 1 A $2 \times 2$ cross-tabulation table for calculation of diagnostic tests.

\begin{tabular}{lccc}
\hline Diagnostic test & \multicolumn{2}{c}{ Gold standard test } & Row total \\
\cline { 2 - 3 } & Positive & Negative & \\
\hline Positive & TP & FP & TP + FP \\
Negative & FN & TN & FN + TN \\
Column Total & TP + FN & FP + TN & \\
\hline
\end{tabular}

${ }^{\dagger} \mathrm{TP}=$ true positive, $\mathrm{FP}=$ false positive, $\mathrm{TN}=$ true negative, $\mathrm{FN}=$ false negative.

were analysed on a $2.0 \%$ AGE stained with ethidium bromide and observed under a UV transilluminator.

\section{Validity of LAMP-MG assay}

Validity of the LAMP-MG assay for CMhm was determined against the PCR assay. The percentage of sensitivity, specificity, positive predictive value (PPV), negative predictive value (NPV), prevalence, and accuracy were calculated in format of $2 \times 2$ cross-tabulation table (Table 1 ) using MEDCALC (www.medcalc.org/calc/diagnostic_test.php). Cohen's kappa coefficient $\kappa$ was calculated to assess the degree of agreement between two diagnostic tests using VASSARSTATS for statistical computation (www.vassarstats.net/kappa.html). All statistical analyses were calculated within $95 \%$ confidence intervals (CIs).

\section{RESULTS}

\section{Optimization of LAMP-MG}

The data revealed that the reaction temperatures at 61,63 , and $65^{\circ} \mathrm{C}$ had no difference of LAMP amplification efficiency (Fig. 2a). Hence the intermediate temperature at $63^{\circ} \mathrm{C}$ was chosen to perform every LAMP-MG test in this study. The optimum concentration of MG dye indicated that $0.004 \%$ MG was suitable for discrimination of the results as light blue and colourless in positive and negative reactions, respectively (Fig. 2b).

\section{Limit of detection of LAMP-MG assay}

The detection limits of LAMP-MG, as well as standard LAMP-turbidity assays, were $2.64 \times 10^{2}$ copies corresponding to $2.64 \times 10^{2}$ organisms/ $\mu$ l of $\mathrm{CMhm}$ feline blood (Fig. 3a-c). In addition, LOD of standard PCR revealed the similar sensitivity to those of LAMP assays corresponding to $2.64 \times 10^{2}$ organisms/ $\mu$ l of CMhm feline blood (Fig. 4).

\section{Clinical sample tests}

A total of 105 clinical samples were evaluated by LAMP-MG assay comparing with PCR as gold stan- 


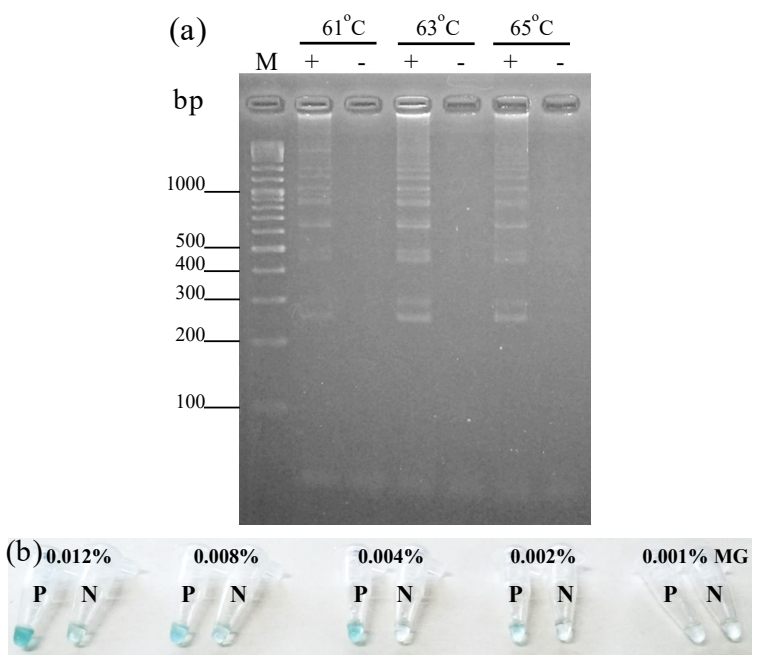

Fig. 2 Optimization of LAMP-MG assay: (a) a 2.0\% AGE profile of the reaction temperatures at 61,63 , and $65^{\circ} \mathrm{C}$ for $60 \mathrm{~min}$, lane M represents GeneRuler DNA Ladder mix (100-10000 bp) marker (Thermo Scientific, USA), and (b) MG concentration tests at $0.012 \%, 0.008 \%, 0.004 \%$, $0.002 \%$, and $0.001 \%$ at $63{ }^{\circ} \mathrm{C}$ for $60 \mathrm{~min}$. $\mathrm{P}$ and $\mathrm{N}$ represent positive and negative reactions, respectively.

dard test (Table 2). Using the LAMP-MG assay, 17 and 88 samples were $\mathrm{CMhm}$ positive and negative, respectively; whereas using the PCR assay, 15 and 90 samples were $\mathrm{CMhm}$ positive and negative, respectively. The results of DNA sequencing revealed that the positive DNA amplified products were $98 \%$ identity to $\mathrm{CMhm}$.

\section{Validity of LAMP-MG assay}

The validity of the LAMP-MG assay was calculated against PCR as gold standard test. The data demonstrated that the sensitivity $=100 \%$ (CI: 78.2$100.0 \%$ ), specificity $=97.8 \%$ (CI: 92.2-99.7\%), $\mathrm{PPV}=88.2 \%$ (CI: 63.6-98.5\%), NPV $=100.0 \%$ (CI: 95.9-100\%), prevalence $=14.3 \%$ (CI: 8.2-22.5\%), and accuracy $=98.1 \%$ (CI: 92.6-99.7\%). The degree of agreement between the LAMP-MG and PCR assays was $92.6 \%, \kappa=1$ (CI: 82.5-100.0\%).

Table 2 Data interpretation of clinical sample tests.

\begin{tabular}{lccc}
\hline LAMP-MG & \multicolumn{2}{c}{ PCR } & Total \\
\cline { 2 - 3 } & + & - & \\
\hline+ & 15 & 2 & 17 \\
- & 0 & 88 & 88 \\
Total & 15 & 90 & 105 \\
\hline
\end{tabular}

(a)

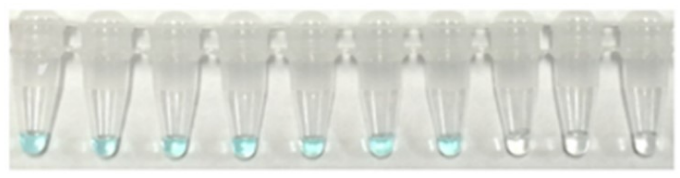

(b)

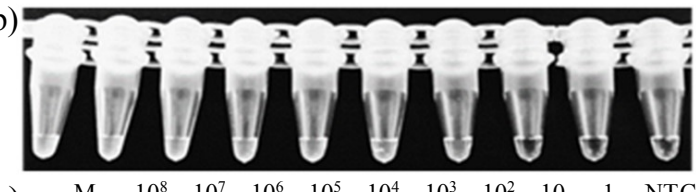

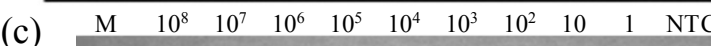

$\mathrm{bp} \Leftrightarrow \boxminus \boxminus \boxminus \boxminus \boxminus \boxminus \boxminus E$

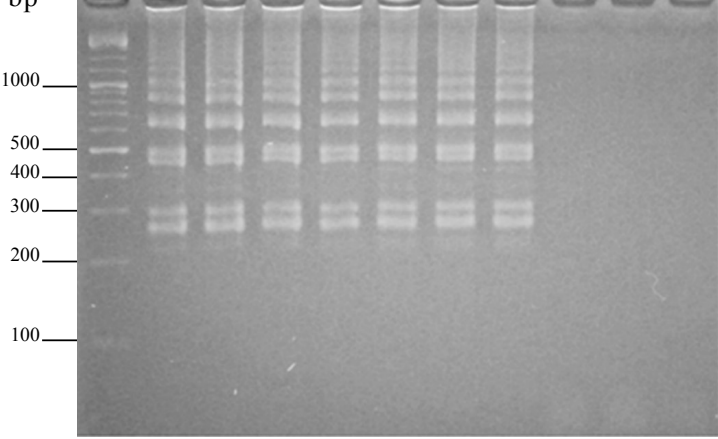

Fig. 3 Detection limit of (a) LAMP-MG assay, (b) standard LAMP-turbidity, and (c) LAMP-2.0\% AGE; lane M represents GeneRuler DNA Ladder mix (100-10000 bp) marker (Thermo Scientific, USA) by using a ten-fold serial dilution of $16 \mathrm{~S}$ CMhm recombinant plasmid DNA $\left(10^{8}\right.$ to 1 copies). NCT represents no template control.

\section{DISCUSSION}

With an increasing number of stray cats in Thailand since 2005 as reported by Department of Communicable Disease Control, FIA has become an emerging infectious disease that can be transmitted among stray and domestic cats. Hence asymptomatic domestic cats that intend to be blood donors can be carriers for the disease. Thus the early detection of CMhm infection is required and essential for the screening of blood donors and controlling FHM in endemic areas. Herein, an LAMP-MG colorimetric assay has been developed as a rapid, sensitive and specific tool for the detection of CMhm based on the 16S rRNA gene due to its uniqueness in individual specific sequence species with a single copy gene and distinct size from Mhf and CMtc. Furthermore, studies of FHM infection based on 16S rRNA gene coupled with internal control as feline 28S rRNA gene $^{33}$, or feline glyceraldehyde-3-phosphate dehydrogenase (FG3PDH) gene ${ }^{34}$ have been reported. While FHM sequence information on other genes need to be further investigated.

Using of MG as $\mathrm{pH}$-sensitive indicator dye for 


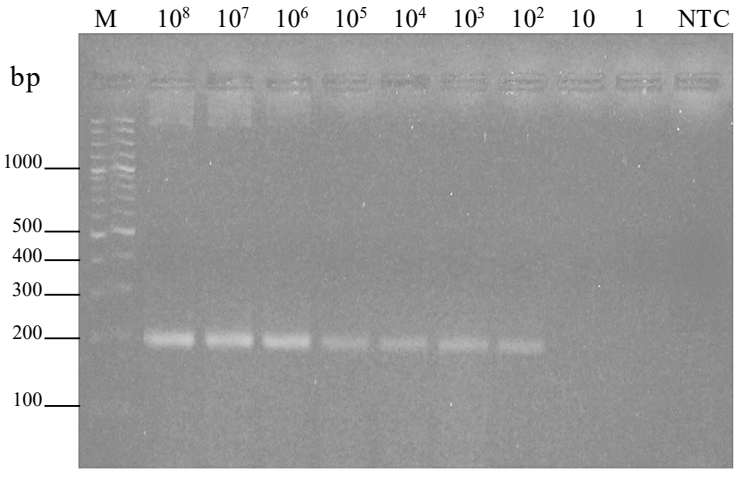

Fig. 4 Detection limit of standard PCR-2.0\% AGE using a ten-fold serial dilution of $16 \mathrm{~S} \mathrm{CMhm}$ recombinant plasmid DNA ( $10^{8}$ to 1 copies), lane $M$ represents GeneRuler 100 bp Plus DNA Ladder (100-3000 bp) marker (Thermo Scientific, USA). NCT represents no template control.

visual end-point assessment of LAMP products in various infections caused by bacteria ${ }^{26}$ and protozoa $^{27,28,35}$ have been reported, however it has not yet been studied in FHM infection especially in CMhm agent. The role of MG in LAMP reaction starting under alkaline condition, in positive reaction, LAMP progressed by producing large amount of DNA product resulting in increasing acidic condition. Furthermore, by product pyrophosphate ions were produced and reacted to magnesium ions forming an insoluble white precipitate of magnesium pyrophosphate making the solution slightly shifted from initial dark blue to light blue (pH 3-9). Whereas in negative reaction, the solution remained alkaline condition and represented as transparent colour $(\mathrm{pH}>10)$. The optimum concentration of MG used throughout this study was at $0.004 \%$, making positive and negative perfectly differentiated. Whereas, higher or lower concentration might lead to judge incorrectly as false positive and false negative, respectively.

LOD of the LAMP-MG and standard LAMPturbidity assays were both $2.64 \times 10^{2}$ copies corresponding to $2.64 \times 10^{2}$ organisms $/ \mu$ l of CMhm feline blood. This finding suggested that the pre-addition of MG did not interfere with the efficiency of LAMP. LAMP can work well in the presence of inhibitors, such as EDTA and other substances in blood, which cannot always be removed during DNA purification. To enhance the sensitivity of detection, the addition of loop primers and extension of the amplification time may be required. According to the LAMPMG, positive and negative reactions can be simply judged by the naked eye on different shades of colour as light blue and colourless. In contrast, standard LAMP-turbidity requires the observation on white precipitate which may not give compatible results due to variation in eyesight of individuals ${ }^{36}$ and its rapid disintegration ${ }^{24}$. However, a higher costly tool as real-time turbidimeter might be used to diminish the limitations.

LAMP-MG was applied for the detection of CMhm in 105 clinical EDTA blood samples against PCR as a gold standard test. Upon comparison, two samples were positive with LAMP-MG but negative with PCR. However, the LOD of standard PCR revealed the same result to LAMP-MG at $2.64 \times 10^{2}$ copies. It is possible that these two samples might not be perfectly cleaned during DNA purification and hindered by inhibitors. However, LAMP itself has a higher tolerance to inhibitors than that of $\mathrm{PCR}^{37}$ with excess magnesium concentration presented in the reaction. Besides, these two samples might contain very low bacterial density that was undetectable by PCR, whereas it was partially detected by the LAMP-MG assay (weakly positive). However, because of the limit of clinical sample amounts, it was not possible to perform additional runs.

We also tested our assay specificity with canine bacterial parasites and haemoflagellates (Anaplasma platys, Ehrlichia canis, and Trypanosoma evansi), with satisfying results. However, our assay did not test another organism in the genus Mycoplasma spp. The nucleotide sequence analysis using the BLAST showed that no Mycoplasma spp. were related to our primer sequences (data not shown). Based on the statistical analysis of LAMP-MG and PCR assays, there was an agreement in diagnostic sensitivity ( $\kappa=1$; CI: $82.5-100.0 \%$ ) with $100.0 \%$ sensitivity (CI: 78.2-100.0\%), 97.8\% specificity (CI: 92.299.7\%) and $98.1 \%$ accuracy (CI: $92.6-99.7 \%$ ), respectively. Furthermore, LAMP-MG assay can be accomplished with an enormous number of samples within $60 \mathrm{~min}$ and simply judged on colour either light blue or colourless by naked eye at once amplification was completed. LAMP-MG did not require any special or costly equipment, such as turbidimeter or dangerous UV illuminator. LAMPMG is a label free technique that can be completed in a single tube-closed system, thus contamination from any environment is diminished. In addition, MG can be stored at room temperature without loss of activity and generates a stable colour after amplification supporting for the record purpose ${ }^{30}$. Hence LAMP-MG is appropriate to be used as a rapid screening testing for the early detection of $\mathrm{CMhm}$ 
infection worldwide.

In conclusion, the present study demonstrates that we developed the LAMP-MG assay for the rapid detection of $\mathrm{CMhm}$ infection in cats. Limit of detection of LAMP-MG was equal to standard LAMP-turbidity and PCR assays corresponding to $2.64 \times 10^{2}$ organisms $/ \mu 1$ of $\mathrm{CMhm}$ feline blood. Outstandingly, LAMP-MG did not hinder DNA synthesis activity and maintained good reaction even in presenting of inhibitors. Further, LAMP-MG can be easily performed as well as simply detected by naked eye given satisfying results as robustness, high sensitivity, specificity and accuracy within $60 \mathrm{~min}$ of reaction time. This technique can be used as alternative method for detection of feline CMhm infection.

Acknowledgements: This work was financially supported by the Thailand Research Fund through the Royal Golden Jubilee Ph.D. Programme under Grant No. PHD/0003/2553 and co-financed by the Higher Education Research Promotion under Grant No. 140/2559 and the Graduate School of Srinakharinwirot University, Thailand.

\section{REFERENCES}

1. Willi B, Boretti FS, Tasker S, Meli ML, Wengi N, Reusch CE, Lutz H, Hofmann-Lehmann R (2007) From Haemobartonella to hemoplasma: molecular methods provide new insights. Vet Microbiol 125, 197-209.

2. Flint JC, Moss LC (1953) Infectious anemia in cats. $J$ Am Vet Med Assoc 122, 45-8.

3. Foley JE, Pedersen NC (2001) Candidatus Mycoplasma haemominutum, a low-virulence epierythrocytic parasite of cats. Int J Syst Evol Microbiol 51, 815-7.

4. Cooper SK, Berent LM, Messickv JB (1999) Competitive, quantitative PCR analysis of Haemobartonella felis in the blood of experimentally infected cats. $J$ Microbiol Meth 34, 235-44.

5. Inokuma $H$, Taroura $S$, Okuda $M$, Hisasue $M$, Itamoto K, Une S, Nakaichi M, Taura Y (2004) Molecular survey of Mycoplasma haemofelis and 'Candidatus Mycoplasma haemominutum' infection in cats in Yamaguchi and surrounding areas. J Vet Med Sci 66, 1017-20.

6. Chungpivat S, Sarikaputi M, Jirasukprasert S, Jenchangkol S, Viseshakul N (2007) The molecular identification of Mycoplasma haemofelis and Mycoplasma haemominutum in cats suffering from hemoplasmosis in Thailand. Thai $J$ Vet Med 37, 33-40.

7. Bauer N, Balzer HJ, Thüre S, Moritz A (2008) Prevalence of feline hemotropic mycoplasmas in convenience samples of cats in Germany. J Feline Med Surg 10, 252-8.
8. Peters IR, Helps CR, Willi B, Hofmann-Lehmann R, Tasker S (2008) The prevalence of three species of feline haemoplasmas in samples submitted to a diagnostics service as determined by three novel realtime duplex PCR assays. Vet Microbiol 126, 142-50.

9. Jiyipong T, Amavisit P, Tasker S, Peters I, Stich RW, Jittapalapong S (2008) Molecular detection of feline hemoplasma in stray cats in Bangkok, Thailand. Infect Genet Evol 8, S21-2.

10. Jiyipong T, Amavisit P, Wongnarkpet S, Tasker S, Jittapalapong S (2008) Molecular epidemiology of Mycoplasma haemofelis and 'Candidatus Mycoplasma haemominutum' of stray cats in Bangkok. In: Proceedings of the 15th Congress of FAVA, Bangkok, Thailand, pp O23-4.

11. Tasker S, Braddock JA, Baral R, Helps CR, Day MJ, Gruffydd-Jones TJ, Malik R (2004) Diagnosis of feline haemoplasma infection in Australian cats using a real-time PCR assay. $J$ Feline Med Surg 6, 345-54.

12. Lappin MR, Griffin B, Brunt J, Riley A, Burney D, Hawley J, Brewer MM, Jensen WA (2006) Prevalence of Bartonella species, haemoplasma species, Ehrlichia species, Anaplasma phagocytophilum, and Neorickettsia risticii DNA in the blood of cats and their fleas in the United States. J Feline Med Surg 8, 85-90.

13. Shaw SE, Kenny MJ, Tasker S, Birtles RJ (2004) Pathogen carriage by the cat flea Ctenocephalides felis (Bouche) in the United Kingdom. Vet Microbiol 102, 183-8.

14. Woods JE, Brewer MM, Hawley JR, Wisnewski N, Lappin MR (2005) Evaluation of experimental transmission of Candidatus Mycoplasma haemominutum and Mycoplasma haemofelis by Ctenocephalides felis to cats. Am J Vet Res 66, 1008-12.

15. Tasker S, Lappin MR (2002) Haemobartonella felis: recent developments in diagnosis and treatment. $J$ Feline Med Surg 4, 3-11.

16. Alleman AR, Pate MG, Harvey JW, Gaskin JM, Barbet AF (1999) Western immunoblot analysis of the antigens of Haemobartonella felis with sera from experimentally infected cats. J Clin Microbiol 37, 1474-9.

17. Wolf-Jäckel GA, Jäckel C, Museux K, Hoelzle K, Tasker S, Lutz H, Hofmann-Lehmann R (2010) Identification, characterization, and application of a recombinant antigen for the serological investigation of feline hemotropic Mycoplasma infections. Clin Vaccine Immunol 17, 1917-25.

18. Jensen WA, Lappin MR, Kamkar S, Reagan WJ (2001) Use of a polymerase chain reaction assay to detect and differentiate two strains of Haemobartonella felis in naturally infected cats. Am J Vet Res 62, 604-8.

19. Berent LM, Messick JB, Cooper SK (1998) Detection of Haemobartonella felis in cats with experimentally induced acute and chronic infections, using a polymerase chain reaction assay. Am J Vet Res 59, 1215-20. 
20. Suksai P, Sangkachai N, Chatsiriwech J, Kanthasaewee O, Sariya L, Chaichoun K (2010) Development of multiplex polymerase chain reaction for detection of feline hemotropic mycoplasma in blood and tissue specimens. Southeast Asian J Trop Med Publ Health 41, 1447-53.

21. Tasker S, Helps CR, Day MJ, Gruffydd-Jones TJ, Harbour DA (2003) Use of real-time PCR to detect and quantify Mycoplasma haemofelis and Candidatus Mycoplasma haemominutum DNA. J Clin Microbiol 41, 439-41.

22. Lobetti RG, Tasker S (2004) Diagnosis of feline haemoplasma infection using a real-time PCR assay. J S Afr Vet Assoc 75, 94-9.

23. Notomi T, Okayama H, Masubuchi H, Yonekawa T, Watanabe K, Amino N, Hase T (2000) Loop-mediated isothermal amplification of DNA. Nucleic Acids Res 28, e63.

24. Almasi MA, Ojaghkandi MA, Hemmatabadi A, Hamidi F, Aghaei S (2013) Development of colorimetric loop-mediated isothermal amplification assay for rapid detection of the Tomato Yellow Leaf Curl virus. J Plant Pathol Microbiol 4, 153.

25. Tanner NA, Zhang Y, Evans TC Jr (2015) Visual detection of isothermal nucleic acid amplification using pH-sensitive dyes. BioTechniques 58, 59-68.

26. Farnia P, Masjedi MR, Mohammadi F, Tabarsei P, Farnia P, Mohammadzadeh AR, Baghei P, Varahram M, et al (2008) Colorimetric detection of multidrugresistant or extensively drug-resistant tuberculosis by use of malachite green indicator dye. $J$ Clin Microbiol 46, 796-9.

27. Nzelu CO, Cáceres AG, Guerrero-Quincho S, TineoVillafuerte E, Rodriquez-Delfin L, Mimori T, Uezato H, Katakura K, et al (2016) A rapid molecular diagnosis of cutaneous leishmaniasis by colorimetric malachite green-loop-mediated isothermal amplification (LAMP) combined with an FTA card as a direct sampling tool. Acta Trop 153, 116-9.

28. Lucchi NW, Ljolje D, Silva-Flannery L, Udhayakumar V (2016) Use of malachite green-loop mediated isothermal amplification for detection of Plasmodium spp. parasites. PLoS One 11, e0151437.

29. Elaziouti A, Laouedj N, Ahmed B (2011) Effect of $\mathrm{pH}$ solution on the optical properties of cationic dyes in dye/Maghnia montmorillonite suspensions. $J$ Chem Eng Process Tech 2, 113-7.

30. Nzelu CO, Gomez EA, Cáceres AG, Sakurai T, MartiniRobles L, Uezato $\mathrm{H}$, Mimori T, Katakura K, et al (2014) Development of a loop-mediated isothermal amplification method for rapid mass-screening of sand flies for Leishmania infection. Acta Trop 132, $1-6$.

31. Guimaraes AM, Santos AP, do Nascimento NC, Timenetsky J, Messick JB (2014) Comparative genomics and phylogenomics of hemotrophic mycoplasmas. PLoS One 9, e91445.
32. Kewish KE, Appleyard GD, Myers SL, Kidney BA, Jackson ML (2004) Mycoplasma haemofelis and Mycoplasma haemominutum detection by polymerase chain reaction in cats from Saskatchewan and Alberta. Can Vet $J$ 45, 749-52.

33. Aquino LC, Hicks CAE, Scalon MC, Lima MGM, Lemos MS, Paludo GR, Helps CR, Tasker S (2014) Prevalence and phylogenetic analysis of haemoplasmas from cats infected with multiple species. $J \mathrm{Mi}$ crobiol Meth 107, 189-96.

34. Watanabe M, Hisasue M, Souma T, Ohshiro S, Yamada T, Tsuchiya R (2008) Molecular detection of Mycoplasma haemofelis and 'Candidatus Mycoplasma haemominutum' infection in cats by direct PCR using whole blood without DNA extraction. $J$ Vet Med Sci 70, 1095-9.

35. Sriworarat C, Phumee A, Mungthin M, Leelayoova S, Siriyasatien P (2015) Development of loop-mediated isothermal amplification (LAMP) for simple detection of Leishmania infection. Parasites Vectors 8, 591.

36. Sappat A, Jaroenram W, Puthawibool T, Lomas T, Tuantranont A, Kiatpathomchai W (2011) Detection of shrimp Taura syndrome virus by loop-mediated isothermal amplification using a designed portable multi-channel turbidimeter. $J$ Virol Meth 175, 141-8.

37. Aryan E, Meshkat Z, Mirbagheri SZ, Alvandi AH, Safdari H, Riahi B, Momen-Heravi M (2014) Improved detection of mycobacterium tuberculosis using an inhibitor-tolerant loop-mediated isothermal amplification. Eur Respir J 44, Suppl 58, P2588. 